\title{
Conciliación y salud laboral: ¿una relación posible? Actualidad en el estudio del conflicto trabajo-familia y la recuperación del estrés
}

\author{
Work-family balance and occupational health: Is this relationship possible? $\mathrm{Cu}$ - \\ rrent situation in the study of work-family conflict and recovery from stress
}

\section{Ana Isabel Sanz Vergel}

IE University, Segovia

Correspondencia:

Ana Isabel Sanz Vergel

IE University

Campus de Santa Cruz la Real

Cardenal Zúñiga, 12

40003 Segovia (España)

Tfno.: +34921412410

E-mail: aisanz@faculty.ie.edu

Resumen

Los cambios económicos y sociales de los últimos años han generado nuevas estructuras organizacionales y familiares que afectan a los trabajadores. Aparecen así nuevos riesgos psicosociales, como el conflicto entre la vida laboral y familiar. Para poder enfrentarse a las demandas del trabajo y de la familia, es necesario tener un tiempo para recuperarnos del esfuerzo realizado. Así pues, para mejorar la calidad de vida del empleado, es necesario atender al bienestar de la persona en su conjunto.

El objetivo del presente artículo es revisar las principales líneas de investigación surgidas en las últimas décadas en torno a estos temas. En primer lugar, se analizan los factores antecedentes del conflicto trabajofamilia así como sus consecuencias y diversas variables que intervienen en este proceso. Posteriormente, se examina la literatura reciente sobre "recuperación del estrés", atendiendo a las estrategias que pueden ayudar a los trabajadores a superar los esfuerzos llevados a cabo en la rutina diaria. Por último, se presentan las principales líneas de prevención e intervención a nivel organizacional e individual, dirigidas a reducir los estresores laborales, promover políticas de conciliación y desarrollar estrategias de recuperación.

Palabras clave: Conflicto trabajo-familia, recuperación del estrés, politicas de conciliación, estrategias de recuperación.

\begin{abstract}
The economic and social changes of the last years have created new organizational and family structures that affect workers. As a result, new psychosocial risks appear, as the conflict between work and family life. To meet work and family demands, people need time to recover from the expended effort. Thus, to improve the quality of life of the employee, it is necessary to attend to the well-being of the person in general.

The aim of this paper is to review the main lines of research emerged during the last decades on these topics. First, we analyze the background and consequences of work-family conflict as well as the main variables affecting this process. Second, we examine recent literature on "recovery from stress", focusing on the strategies that may help workers to overcome the efforts made during the daily routine. Finally, we present the main lines of prevention and intervention in the organizational and individual levels, aimed at reducing job stressors, promoting family-friendly policies and developing recovery strategies.
\end{abstract}

Key words: Work-family conflict, recovery from stress, family-friendly policies, recovery strategies. 


\section{INTRODUCCIÓN}

Las relaciones entre el trabajo y la familia articulan el día a día de los trabajadores, de modo que su estudio dentro del campo de la Salud Laboral es fundamental. De hecho, Moreno-Jiménez y Báez ${ }^{1}$ señalan que uno de los riesgos psicosociales que más atención está recibiendo en los últimos años es precisamente el conflicto trabajo-familia. La aparición de estos nuevos riesgos es resultado de una serie de cambios tanto a nivel económico como social. Los largos horarios de trabajo en respuesta a la competitividad empresarial, o la incorporación de la mujer al mercado laboral, generan nuevas estructuras organizacionales y también familiares, que afectan al trabajador. Un estudio realizado en 2005 por el Instituto de la Mujer, refleja que el $51 \%$ de las mujeres y el $40 \%$ de los hombres consideran que el equilibrio entre la esfera laboral y familiar resulta complicado ${ }^{2}$.

A nivel social, la preocupación por el problema de la conciliación ha quedado patente en la Ley Orgánica de Igualdad entre mujeres y hombres (BOE n. 71 de 23/3/2007), que en su artículo 44.1 refleja que "los derechos de conciliación de la vida personal, familiar y laboral se reconocerán a los trabajadores y trabajadoras en forma que fomenten la asunción equilibrada de las responsabilidades familiares, evitando toda discriminación basada en su ejercicio"”.

Lo cierto es que cuando queremos mejorar la calidad de vida de los trabajadores, no podemos olvidar que estas personas son también padres, esposos, amigos, o hijos. Tienen una vida personal más allá de las exigencias laborales, y por ello hay que atender al bienestar de la persona en su conjunto. Por lo tanto, poder conciliar el trabajo y la familia es un indicador de salud laboral. La cuestión es: ¿es esto posible? ¿Cómo puede ayudar la organización a compaginar estos dos ámbitos? ¿Qué estrategias puede utilizar la persona para recuperarse de las demandas diarias?

El objetivo de este artículo es revisar las principales líneas de investigación surgidas en las últimas décadas en torno a estos temas, analizando los principales factores antecedentes del conflicto trabajo-familia y sus consecuencias. Así mismo, se examinará la literatura reciente sobre "recuperación del estrés", atendiendo a las principales estrategias que pueden ayudar a los trabajadores a superar los esfuerzos llevados a cabo en la rutina diaria.

\section{LA CONCILIACIÓN ENTRE LA VIDA LABORAL Y FAMILIAR: LA APARICIÓN DEL CONFLICTO}

El origen de este campo de investigación se remonta a finales de los años 70 con el trabajo de Pleck ${ }^{4}$, en el que se reconoce que el trabajo y la familia se influyen mutuamente a partir de las ideas, emociones, y actitudes surgidas en cada uno de estos dominios. Aunque se han empleado diversos términos para referirse a las relaciones existentes entre el trabajo y la familia (p.e. interferencia trabajo-familia, interacción trabajo-familia), el más ampliamente propuesto ha sido el concepto de "conflicto trabajo-familia", definido por Greenhaus y Beutell ${ }^{5}$ como "una forma de conflicto de rol, en el que las presiones que resultan del trabajo y las presiones familiares son mutuamente incompatibles en algún aspecto" (p.77).

Esta definición, continúa siendo un punto de referencia en las investigaciones sobre conciliación vida laboral y familiar, ya que enfatiza un aspecto fundamental para la comprensión de estos dominios: la bidireccionalidad existente entre el trabajo y la familia. A partir de esta idea, se han identificado dos tipos de conflicto: uno en el que el trabajo interfiere en la familia, y otro en el que la familia interfiere en el trabajo ${ }^{6}$. En concreto, el conflicto trabajo-familia ocurre cuando las tareas laborales interfieren con las familiares, como el hecho de tener que llevar trabajo a casa alterando la dinámica y la interacción familiar. Por otra parte, el conflicto familia-trabajo tiene lugar cuando el desempeño de responsabilidades familiares obstaculiza las tareas laborales, como el tener que cancelar 
una reunión porque un hijo se ha puesto enfermo. Se trata de dos procesos diferentes, cada uno de ellos con sus propios mecanismos específicos.

Los investigadores están interesados en examinar ambos dominios y cómo las experiencias en un dominio afectan al otro. Sin embargo, la mayoría de estudios se han centrado en la dirección trabajo-familia, principalmente porque suele ser el tipo de conflicto que se produce con mayor frecuencia. Los muros entre la vida laboral y familiar parecen ser asimétricamente permeables, es decir, con frecuencia dejamos que los problemas en el trabajo afecten a nuestra vida familiar, sin embargo, cuando tenemos un problema relacionado con nuestra vida privada, hacemos todo lo posible por dejarlo a un lado y que éste no interfiera en nuestras responsabilidades laborales ${ }^{7}$.

Para entender mejor el fenómeno del conflicto trabajo-familia, es necesario atender a los factores que dan origen al problema, así como a los efectos que produce. Además, este análisis se debe realizar desde una perspectiva transaccional, considerando la experiencia de conflicto como un proceso en el que intervienen diversas variables que afectan la fuerza de las relaciones entre los antecedentes, el conflicto, y las consecuencias. Este proceso es el que se analiza en los siguientes apartados.

\section{Factores antecedentes del conflicto trabajo-familia}

Numerosos estudios se han centrado en analizar las relaciones entre distintas demandas y ambos tipos de conflicto. Puesto que las relaciones entre el trabajo y la familia son bidireccionales, es necesario identificar también dos tipos de demandas con el objetivo de esclarecer las conexiones entre estos dos ámbitos: las demandas laborales y las demandas familiares. El interés por examinar los antecedentes de ambos tipos de conflicto, se ha apoyado generalmente en la teoría del rol, desarrollada por Kahn y colaboradores $^{8}$, convirtiéndose en una de las más extendidas en este campo de investigación.

El argumento central de esta teoría es que las personas desempeñan diferentes roles que se corresponden con determinadas expectativas. Sin embargo, cuando las personas se enfrentan a diversas demandas, teniendo que desempeñar múltiples roles a la vez, resulta muy complicado cumplir las expectativas de todos esos roles, por lo que es probable que se experimente conflicto. Por ejemplo, cuando tienen que desempeñar el rol de padres y de trabajadores, pueden surgir conflictos, ya que éstos pueden ser incompatibles en muchos momentos.

Se ha demostrado que los mejores predictores del conflicto trabajo-familia son las demandas laborales, mientras que los mejores predictores del conflicto familia-trabajo son las demandas familiares ${ }^{9}$. De hecho, en un reciente meta-análisis basado en 1080 correlaciones procedentes de 178 muestras, se vuelve a reflejar este patrón ${ }^{10}$.

En cuanto a las demandas laborales, la más ampliamente estudiada ha sido el número de horas de trabajo. Diversas investigaciones han demostrado que cuantas más horas trabaja una persona, mayores niveles de conflicto trabajo-familia experimenta ${ }^{11}$. El trabajo a turnos y en horario nocturno también dificulta la conciliación entre la vida laboral y familiar. Este tipo de jornadas afecta las relaciones personales de los trabajadores e interfieren en el desempeño de sus actividades domésticas ${ }^{12}$.

Con respecto a otros estresores, los resultados del estudio de Boyar, Maertz Jr., Pearson y Keough ${ }^{13}$ muestran que la sobrecarga y el conflicto de rol se asocian de forma positiva con el conflicto trabajo-familia. En España, Marín, Infante y Rivero ${ }^{14}$ encontraron que el principal predictor del conflicto trabajo-familia era la sobrecarga, seguida de la ambigüedad y el conflicto de rol.

Pero tal como se ha comentado previamente, las demandas laborales no son las únicas que pueden conducir a un conflicto, puesto que éste es bidireccional, las demandas familiares también pueden obstaculizar el desempeño de las tareas laborales. En general, las demandas familiares más estudiadas se relacionan con la estructura familiar, es decir, 
si la persona convive con su pareja, si tienen hijos, o si hay personas mayores a su cargo $^{15}$. De estas demandas, parece que la carga que supone el cuidado de los hijos, es la que conduce a mayores niveles de conflicto familia-trabajo ${ }^{16}$.

\section{Las consecuencias del conflicto trabajo-familia}

El conflicto trabajo-familia ha sido considerado en sí mismo como una fuente de estrés que tiene una serie de consecuencias a nivel organizacional y familiar. En un metaanálisis realizado en 2007 por Ford, Heinen y Langkamer ${ }^{17}$, se ha encontrado que cada tipo de conflicto conduce a efectos negativos en el otro dominio (por ejemplo, el conflicto trabajo-familia conduce a insatisfacción familiar, mientras que el conflicto familia-trabajo conduce a insatisfacción laboral). Sin embargo, algunos autores han encontrado que también pueden existir efectos dentro del mismo dominio.

Por ejemplo, el conflicto trabajo-familia también puede conducir a menores niveles de satisfacción laboral ${ }^{18}$, así como a mayor intención de abandonar la empresa ${ }^{15}$. Un punto clave dentro del estudio de las consecuencias del conflicto, es analizar su efecto sobre la salud y el bienestar del trabajador. En este caso, las investigaciones realizadas parecen coincidir en que ambos tipos de conflicto se asocian con mayores niveles de tensión psicológica ${ }^{19}$

Por su parte, Lapierre y Allen ${ }^{20}$ han distinguido entre bienestar afectivo y bienestar físico. El bienestar físico hace referencia exclusivamente a síntomas somáticos (p.e. fatiga, dolor de cabeza, náuseas), mientras que el bienestar afectivo se relaciona con emociones negativas como ansiedad, depresión o irritabilidad. Estos autores encontraron una relación negativa entre estos dos tipos de bienestar y el conflicto trabajo-familia.

Por lo tanto, cuando las energías empleadas en el trabajo te impiden dedicarte a tus responsabilidades familiares, es más probable que se experimenten síntomas físicos y emociones negativas. Por su parte, Mauno, Kinnunen y Pyykkö ${ }^{21}$ distinguieron tres dimensiones del bienestar: síntomas físicos, agotamiento (para referirse a síntomas de carácter emocional) y estado de ánimo negativo en el trabajo. Los resultados demuestran que el conflicto trabajo-familia se relaciona positivamente con estas tres dimensiones.

Por último, otra variable ampliamente estudiada ha sido el bienestar subjetivo de la persona. Este término, hace referencia al grado satisfacción que las personas tienen con su vida. La mayoría de estudios que han analizado esta variable, han encontrado que aquellas personas que experimentan altos niveles de conflicto suelen estar menos satisfechas con su vida en general $^{22}$.

\section{VARIABLES MODERADORAS DEL PROCESO DE CONFLICTO TRABAJO-FAMILIA: DE LA ORGANIZACIÓN A LA PERSONA}

El estudio de este proceso quedaría incompleto si no se analizara qué variables pueden estar modificando las relaciones entre las demandas y el conflicto, así como entre el conflicto y sus consecuencias. En este sentido, las investigaciones han tratado de analizar qué puede hacer la organización, qué puede hacer el entorno familiar del trabajador, así como qué variables de la persona pueden influir en el proceso. Estas tres perspectivas son interesantes y tienen implicaciones prácticas para la prevención y la intervención.

\section{A nivel organizacional}

A nivel organizacional, las políticas de conciliación que ofrece una empresa (p.e. flexibilidad de horarios o teletrabajo), se han estudiado como variables moderadoras especialmente entre los estresores laborales y el conflicto trabajo-familia. Por ejemplo, Luk y Shaffer ${ }^{16}$ encontraron que aquellas personas que trabajaban muchas horas pero 
contaban con políticas de conciliación en su empresa, mostraban niveles más bajos de conflicto trabajo-familia.

Además, el apoyo recibido por parte del supervisor ha sido otro objetivo central de estudio. Por ejemplo, un supervisor con el que se puede comentar problemas relacionados con la familia y es flexible cuando surgen problemas relacionados con la vida privada de los empleados, conseguirá hacer que el entorno laboral resulte menos estresante. De hecho, se ha demostrado que dadas las mismas condiciones laborales, existe un nivel más bajo de conflicto trabajo-familia entre aquellas personas que cuentan con el apoyo del supervisor $^{23}$.

Las percepciones del trabajador con respecto al apoyo recibido son también una variable que está atrayendo una gran atención en los últimos años ${ }^{24}$. Así pues, no son suficientes las políticas de conciliación formales, escritas en un papel, sino que la persona tiene que sentir que está recibiendo el apoyo de su supervisor en los asuntos de conciliación trabajo-familia ${ }^{25}$.

\section{A nivel familiar}

Determinadas variables relacionadas con la familia también pueden moderar las relaciones existentes en el proceso de conflicto. En concreto, las variables más estudiadas han sido el apoyo social y el nivel de implicación psicológica en la familia.

Aryee y colaboradores ${ }^{26}$ analizaron el papel del apoyo de la pareja en una muestra de empleados de Hong Kong. Los resultados demostraron que aquellas personas con un gran número de responsabilidades familiares, tenían un menor nivel de conflicto familiatrabajo cuando contaban con el apoyo de sus parejas. En un estudio realizado con mujeres en Malasia se encontró que aquellas mujeres que trabajaban un elevado número de horas, experimentaban menos conflicto trabajo-familia si contaban con el apoyo de sus maridos ${ }^{27}$.

La implicación psicológica en la familia también puede atenuar o exacerbar el nivel de conflicto. Esta variable hace referencia a la importancia que tiene para la persona el dedicarse a sus roles familiares, lo cual puede tener efectos positivos o negativos sobre el conflicto. Por ejemplo, Frone y Rice ${ }^{28}$ encontraron que el nivel de implicación en casa actuaba como una variable moderadora entre el nivel de implicación en el trabajo y el nivel de conflicto trabajo-familia. Lo que estos resultados sugieren es que aquellas personas que están muy implicadas en sus trabajos tienen más dificultades para equilibrar su vida laboral y familiar si además están muy implicadas en sus roles familiares.

\section{Variables personales}

Las características de personalidad han recibido atención en el campo del conflicto trabajo-familia. Este tipo de variables se examinan para demostrar por qué ante los mismos estresores, no todas las personas experimentan el mismo nivel de conflicto. Diversos estudios han encontrado un efecto moderador de ciertas características de personalidad, bien entre los antecedentes y el conflicto, o entre el conflicto y las consecuencias. Por ejemplo, la estabilidad emocional amortigua los efectos negativos del conflicto trabajo-familia sobre el agotamiento y la depresión ${ }^{29}$.

Por último, las estrategias de afrontamiento son sin duda un área importante a explorar. Cuando la persona se enfrenta a una situación de conflicto, trata de poner en marcha una serie de acciones para superar los obstáculos. Pero ¿son esas acciones adecuadas? ¿Ayudan realmente a enfrentarse a la situación? En un estudio llevado a cabo con trabajadores finlandeses, se ha encontrado que la estrategia de afrontamiento centrada en la emoción amortiguaba los efectos de un alto nivel de conflicto familia-trabajo sobre la insatisfacción laboral. Sin embargo, esta misma estrategia, afectaba los niveles de satisfacción familiar ${ }^{30}$.

En general, los estudios llevados a cabo con variables de la persona, muestran resultados a veces incluso contradictorios, que nos deben hacer reflexionar sobre la 
complejidad de este proceso. Como vemos, el número de variables intervinientes es amplio, y están emergiendo nuevos trabajos cuyo objetivo es alcanzar una mayor comprensión de este fenómeno. Entre las líneas más actuales sobre conflicto trabajofamilia y en general sobre la dinámica del estrés laboral, se encuentra un concepto que parece clave para amortiguar los efectos de los estresores. Se trata del concepto de "recuperación del estrés", que trataremos en el siguiente apartado.

\section{ÚLTIMAS LÍNEAS DE INVESTIGACIÓN: EL CONCEPTO DE RECUPERACIÓN}

El concepto de recuperación es definido por Geurts y Sonnentag ${ }^{31}$ como "un proceso de relajación psicofisiológica tras la exposición a una situación que requiere un esfuerzo" (p.485). Desde este punto de vista, la recuperación puede considerarse como un proceso opuesto al proceso de estrés ${ }^{32}$.

En el campo del conflicto trabajo-familia, se han empleado varios de estos términos, sin embargo, ninguno de estos estudios han recogido exactamente la experiencia de recuperación como una variable que interviene en el proceso de conflicto. Por ejemplo, el conflicto trabajo-familia tiene importantes implicaciones para la salud, como el desarrollo de una elevada necesidad de recuperación y un mayor nivel de fatiga ${ }^{33}$. Por su parte, Taris y colaboradores ${ }^{34}$ utilizaron el término de "oportunidades de recuperación", para hacer referencia al hecho de que las personas con altos niveles de conflicto trabajofamilia, tienen menos oportunidades para recuperarse, ya que el esfuerzo invertido en conseguir conciliar estos ámbitos, dificulta o incluso impide esta recuperación. Por tanto, aunque hacen referencia a que el conflicto reduce las oportunidades para recuperarse, no utilizan ninguna medida específica de recuperación. De hecho, en el campo del conflictotrabajo familia, la recuperación aún no se ha considerado como una variable central que puede tener un papel importante en el proceso.

Cuando hablamos de recuperación, en realidad podemos hablar de varios tipos en función de cuándo y cómo ésta tenga lugar. Así pues, podemos realizar una distinción en función de si la recuperación tiene lugar en un contexto laboral, lo cual se conoce como recuperación interna, o en un contexto no laboral, conocido como recuperación externa ${ }^{31}$.

La recuperación interna se refiere a aquellos descansos que se realizan durante la jornada laboral, como una parada para tomar un café o para comer. Estos descansos son importantes para contrarrestar la fatiga y poder mantener un rendimiento adecuado. De hecho, se ha encontrado que la ausencia de estos descansos breves explica al menos parcialmente las altas tasas de estrés entre las personas que trabajan en una oficina ${ }^{35}$. Por otra parte, la recuperación externa es aquella que tiene lugar una vez finalizada la jornada laboral. Este tipo de recuperación es importante porque con frecuencia, la recuperación interna no es suficiente, y es necesario que las personas tengan además la oportunidad de recuperarse tras el esfuerzo realizado en el trabajo, ya sea durante las horas restantes del día, durante los fines de semana, o durante períodos más largos como las vacaciones.

Diversos estudios han demostrado cómo la recuperación externa se relaciona positivamente con el bienestar de los empleados así como con su compromiso laboral y su rendimiento. Por ejemplo, cuando se realizan actividades positivas durante las vacaciones como tomarse tiempo para relajarse o aprender cosas nuevas, posteriormente aumentan los niveles de bienestar y de rendimiento. Sin embargo, si durante este período la persona tiene que enfrentarse a otra serie de situaciones estresantes aunque sea fuera del entorno laboral, aumenta el cansancio, y la persona tiene que realizar un esfuerzo mayor al incorporarse de nuevo a la rutina ${ }^{36}$.

Los efectos negativos de la falta de recuperación también se han encontrado en períodos más cortos de tiempo. De hecho, las experiencias vividas durante el fin de semana $o$ incluso en las horas posteriores a la jornada laboral, pueden ser de gran importancia para mantener el bienestar y un nivel óptimo de rendimiento. 
Por ejemplo, se ha demostrado que aquellas personas que realizan actividades de ocio tras su jornada laboral, al día siguiente muestran mayores niveles de compromiso y son más proactivos en su trabajo ${ }^{37}$.

\section{¿Cómo podemos sentirnos recuperados? La importancia de las experiencias psicológicas subyacentes}

Un punto clave para estudiar la experiencia de la recuperación es comprender por qué determinadas actividades ayudan a la persona a sentirse recuperada. Hasta el momento, los estudios analizan distintos tipos de actividades, pero no las experiencias psicológicas subyacentes. Tal y como Sonnentag y Fritz ${ }^{32}$ proponen, no es una actividad en sí misma la que ayuda a la persona a recuperarse de una situación de estrés, sino que es el proceso psicológico que subyace, como la sensación de relajación o de desconexión, lo que hace que la persona se sienta recuperada. Por tanto, aunque una persona opte por actividades pasivas de ocio, mientras que otra prefiere realizar ejercicio físico, ambas pueden sentirse recuperadas si a través de dicha actividad han conseguido relajarse y renovar sus recursos. Lo importante entonces no es tanto la actividad concreta que se realiza, si no el tener la capacidad de desconectar del trabajo, relajarse, expresar las emociones, en definitiva, "desahogarse" y "renovarse" ya sea a nivel cognitivo, conductual o emocional, para sentirse mejor. Estas autoras proponen cuatro "experiencias" de recuperación que se relacionan con mayores niveles de bienestar psicológico: (a) distanciamiento psicológico del trabajo, (b) relajación, (c) actividades enriquecedoras y (d) control sobre el tiempo libre (ver Tabla I).

Tabla I. Experiencias de Recuperación

\begin{tabular}{ll}
\hline $\begin{array}{l}\text { Distanciamiento } \\
\text { psicológico del trabajo }\end{array}$ & $\begin{array}{l}\text { Alejamiento de la rutina laboral, desde un punto de vista no sólo físico sino } \\
\text { también psicológico. Implica centrarse en otro tipo de actividades distintas } \\
\text { de las desarrolladas en el trabajo, de modo que los sistemas que están activos } \\
\text { mientras la persona se enfrenta a una demanda laboral, puedan permanecer } \\
\text { inactivos por un tiempo }\end{array}$ \\
\hline Relajación & $\begin{array}{l}\text { Se refiere al estado producido por la persona para disminuir de forma } \\
\text { voluntaria su estado de tensión y estrés. Este estado de relajación, se caracteriza } \\
\text { por una baja activación y un elevado afecto positivo }\end{array}$ \\
\hline Búsqueda de retos & $\begin{array}{l}\text { Hace referencia a la realización de actividades que suponen un reto para } \\
\text { la persona y son por tanto un medio para sentirse motivado y buscar la } \\
\text { superación personal. Por ejemplo, aprender un idioma o practicar un nuevo } \\
\text { deporte puede considerarse una forma de recuperación de este tipo ya que } \\
\text { ayuda a adquirir nuevas competencias }\end{array}$ \\
\hline Control sobre el tiempo & $\begin{array}{l}\text { El control se describe como la habilidad de la persona para elegir entre dos o } \\
\text { más opciones. En este caso particular, se refiere al grado en el que una persona } \\
\text { libre }\end{array}$ \\
puede decidir qué actividad llevar a cabo durante su tiempo libre, así como \\
cuándo y cómo llevarla a cabo.
\end{tabular}

De las variables que facilitan la recuperación, la más analizada ha sido el distanciamiento psicológico del trabajo. Por ejemplo, los estudios de diario realizados por el equipo de la Dra. Sonnentag muestran que las personas que se distancian psicológicamente del trabajo tienen mejor humor y experimentan menos fatiga al final del día que aquellos que continúan pensando en asuntos laborales ${ }^{38,39}$.

La investigación reciente está demostrando que la recuperación tiene una conexión importante con el conflicto trabajo-familia. Por ejemplo, se ha encontrado que el distanciamiento psicológico atenúa los efectos negativos del conflicto trabajo-familia sobre la salud ${ }^{40}$. La calidad de sueño, también ejerce un papel amortiguador, reduciendo los efectos negativos del conflicto familia-trabajo sobre la sintomatología psicológica ${ }^{41}$.

Por lo tanto, cabe plantearse un modelo en el que se analicen diversas actividades y estrategias de recuperación, como variables moderadoras en el proceso de conflicto trabajo-familia. En la Figura 1, se proponen una serie de efectos directos de las demandas sobre el conflicto trabajo-familia, que a su vez tiene una serie de consecuencias. Aparte 
de estos efectos directos, lo que interesa especialmente es el análisis de qué puede hacer la persona para recuperarse de la pérdida de recursos que supone el enfrentarse a las demandas laborales y familiares y evitar su impacto negativo en el bienestar.

Figura 1. Modelo del Proceso de Recuperación en el Ámbito del Conflicto Trabajo-Familia.

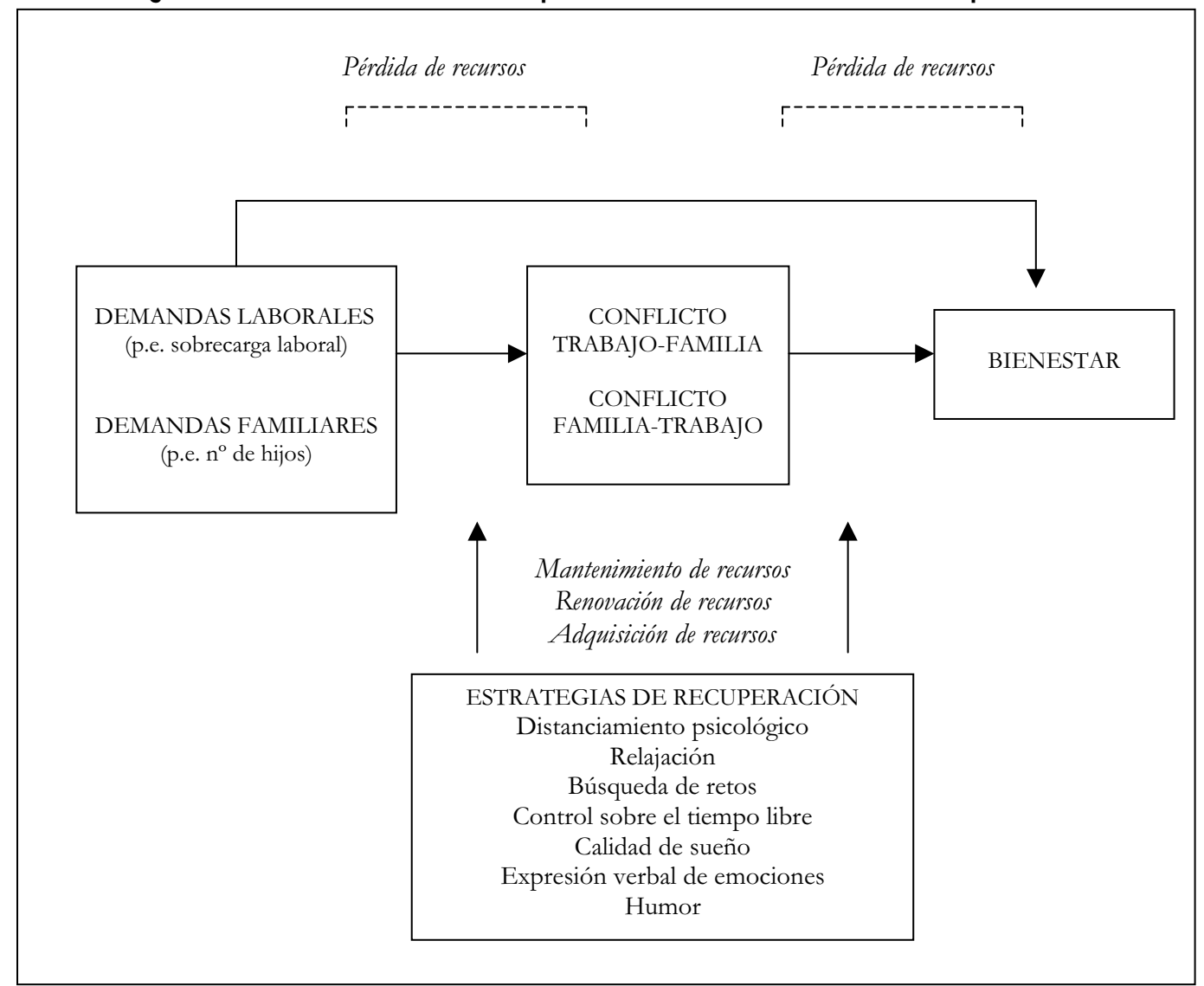

Al ser una línea de investigación incipiente, es importante que las nuevas investigaciones traten de ir más allá, examinando posibles actividades y estrategias que también se puedan considerar dentro del estudio de la recuperación. Así pues, proponemos ampliar el rango de variables que pueden facilitar la recuperación, incluyendo por ejemplo la calidad de sueño, la expresión verbal de emociones, o el humor, entre otras.

Se trata de darle un papel agente y activo a la persona y analizar qué capacidades se pueden desarrollar para que consiga una relación positiva entre su vida laboral y familiar, o al menos para que viva la experiencia de conflicto de la mejor manera posible. Este modelo se enmarca dentro de dos teorías relacionadas con la conciliación trabajofamilia y la recuperación: la Teoría de la Conservación de Recursos de Hobfoll ${ }^{42}$ y el Modelo de Esfuerzo-Recuperación de Meijman y Mulder ${ }^{43}$. La idea central es que, la variedad de actividades y estrategias de recuperación existentes, a través del mantenimiento, la adquisición, y/o la renovación de recursos, podrían amortiguar (1) los efectos negativos de las demandas sobre ambas direcciones del conflicto así como (2) los efectos negativos del conflicto sobre el bienestar.

Por una parte, la Teoría de la Conservación de Recursos argumenta que el conflicto trabajo-familia conduce a problemas de salud debido a los recursos que se pierden tratando de conseguir un equilibrio entre ambos dominios. Además, tiene en cuenta las variables personales como variables importantes que ayudan a reducir los efectos del conflicto. En este caso, el distanciamiento psicológico del trabajo, la expresión verbal de las emociones, o la calidad de sueño, serían formas de recuperar los recursos invertidos e incluso de adquirir otros nuevos. 
Por otra parte, el Modelo de Esfuerzo-Recuperación es útil ya que nos da la base para comprender la importancia de los procesos de recuperación en el contexto de la conciliación trabajo-familia. El estar expuesto a una situación demandante en la que tienes dificultades para atender a todas tus responsabilidades, supone un esfuerzo para la persona. Si no existe un modo de recuperarse de ese esfuerzo para volver a una línea base, la persona entrará en un círculo vicioso en el que cada vez le resultará más complicado enfrentarse a ese conflicto, por lo que finalmente, tendrá mayores problemas de salud y un menor bienestar. Se espera que en el futuro, puedan aparecer diseños más completos que permitan analizar el proceso de conflicto trabajo-familia desde una perspectiva novedosa como es la que ofrece el campo de la recuperación del estrés.

\section{PRINCIPALES LÍNEAS DE PREVENCIÓN E INTERVENCIÓN}

Los resultados de los estudios aquí presentados subrayan la importancia de reducir las demandas e incrementar la recuperación del estrés. Para conseguirlo, es necesario desarrollar intervenciones específicas dirigidas a reducir los estresores, así como ayudar a las personas a desarrollar habilidades y estrategias para enfrentarse a ellos. Así pues, las intervenciones sobre el estrés laboral deben producirse a dos niveles: organizacional e individual. A continuación exponemos las principales líneas de prevención e intervención considerando que la combinación entre ambos niveles es la mejor vía para enfrentarnos a las demandas diarias y mejorar el bienestar.

\section{A nivel organizacional}

Las organizaciones tienen una función capital en el fomento de la conciliación trabajo-familia y de los procesos de recuperación. Se ha reconocido ampliamente que uno de los primeros pasos que las organizaciones deben dar para reducir el nivel de estrés de los trabajadores es un correcto diseño del puesto de trabajo, definiendo claramente las tareas a realizar, ajustando las tareas laborales al tiempo disponible así como a las capacidades del trabajador.

En este sentido, son pilares básicos el desarrollo de políticas de conciliación como los horarios flexibles, o el teletrabajo. Es importante que exista una limitación de los tiempos reales de trabajo, dotando al trabajador con autonomía y apoyándole en las necesidades familiares. Tengamos en cuenta además la importancia de ofrecer la oportunidad de tomarse un descanso durante la jornada laboral, ya que como vimos anteriormente es de vital importancia para reducir el agotamiento. Pero además de las oportunidades de recuperación interna, las organizaciones deberían fomentar la recuperación externa. Por ejemplo, ofertar actividades de ocio o bien organizar actividades sociales o deportivas son algunas de las estrategias que diversas organizaciones ya han empezado a implementar.

Por último, informar y formar a los trabajadores sobre el proceso de estrés y recuperación, dotándoles de herramientas para gestionarlos, es otra práctica que repercutirá positivamente tanto en la empresa como en el trabajador. Algunos de los talleres que se pueden implementar, irían en línea con intervenciones que también se pueden llevar a nivel individual y que comentaremos en la sección siguiente, como el manejo del estrés, la gestión del tiempo de trabajo, o la identificación y planificación de estrategias de recuperación. Por ejemplo, conocer qué están haciendo hasta el momento las personas para intentar recuperarse, e identificar si esto les está resultando realmente útil, es un modo de analizar sus estrategias y poner en práctica aquellas que puedan resultarles más adecuadas en función de su situación.

Para llevar a cabo todas estas medidas, la cultura de la organización debe ser flexible y basada en una comunicación abierta. Esa apertura implica mirar no sólo a la productividad directa sino a los recursos humanos a medio y largo plazo. La capacitación para la 
organización del tiempo laboral y la extensión y desarrollo de un tiempo personal son dos de los grandes medios de que disponen para ello.

\section{A nivel individual}

A pesar de la importancia de tomar medidas a nivel organizacional, centrarse sólo en esta área no siempre es suficiente. De hecho, hay todavía un número elevado de empresas que no atienden a todas estas medidas de forma conjunta, dentro de un plan estratégico más amplio. En los últimos años, debido a la crisis económica, aspectos como las políticas de conciliación, están quedando relegadas a un segundo plano. En otros casos, simplemente no se implementan prácticas de flexibilidad de horarios o reducción de carga de trabajo porque la cultura establecida no reconoce la importancia de dichas medidas. Otro último aspecto a resaltar, es que en otras ocasiones, a esto se une la dificultad de la persona para desarrollar determinadas estrategias de manejo del estrés, o incluso tiene demandas por parte de otras fuentes, como la familia, que hacen aún más difícil atender a todos los estresores. Por eso, es necesario también realizar una prevención e intervención a nivel individual. Dentro de esta planificación, se establecerían objetivos en función de las áreas más problemáticas.

Por ejemplo, para una persona una línea de prevención podría ser la gestión del tiempo, de modo que consiga un control sobre su tiempo libre. Covey, Merril y Merril ${ }^{44}$ propusieron focalizarse en la idea de que no hay que centrarse en cómo hacer más en menos tiempo, sino que lo que te ayuda a organizarte realmente bien, tanto en el trabajo como en tu vida, es identificar lo que realmente te importa. Esto implica una revisión de tus prioridades, y de tus valores, que debe trabajarse como una intervención a nivel individual y no sólo organizacional como podrían ser las tres primeras categorías. Además de la gestión del tiempo, autores como Sonnentag y Bayer ${ }^{38}$ han sugerido diversas técnicas para desconectar psicológicamente, como ejercicios de relajación, o entrenamiento para desarrollar "flow" durante el tiempo libre, es decir, para conseguir que la persona esté inmersa y con la atención focalizada en lo que hace en ese momento.

El objetivo es ayudar a la persona a que desarrolle de modo eficaz estas estrategias. En España, ya se ha enfatizado previamente la necesidad de realizar entrenamiento del manejo del estrés en el ámbito laboral, tanto a nivel individual como a nivel grupal, incluyendo cuatro estrategias: relajación, reestructuración cognitiva, ensayo de habilidades y solución de problemas $^{45}$. La prevención e intervención por tanto, deben realizarse en un marco más amplio en el que la propia sociedad sea consciente de que el bienestar de las personas no es algo que deba solucionarse de forma exclusivamente privada. En el ámbito laboral, las empresas tienen una parte importante de responsabilidad, y desde la Salud Laboral, se intenta fomentar el desarrollo de organizaciones saludables, que en realidad se traduce en la existencia de personas saludables.

\section{REFERENCIAS BIBLIOGRÁFICAS}

1. Moreno-Jiménez B, Báez C. Factores y riesgos psicosociales: Formas, consecuencias, medidas y buenas prácticas. Ministerio de Trabajo e Inmigración. Instituto Nacional de Seguridad e Higiene en el Trabajo. Disponible en: http: // www.insht.es.

2. GPI Consultores. Ministerio de Trabajo y Asuntos Sociales. Instituto de la Mujer. Estudio sobre la conciliación de la vida familiar y la vida laboral: Situación actual, necesidades y demandas. Disponible en: http://www.mtas.es/mujer.

3. Ley Orgánica 3/2007, de 22 de marzo, para la Igualdad efectiva de hombres y mujeres (B.O.E. núm. 071 de 23/03/2007).

4. Pleck JH. The work-family role system. Soc Probl. 1977; 24(4): 417-427.

5. Greenhaus J, Beutell N. Sources of conflict between work and family roles. Acad Manage Rev. 1985; 10(1): 76-88.

6. Frone MR, Russell M, Cooper ML. Antecedents and outcomes of work-family conflict: Testing a model of the work-family interface. J Appl Psychol. 1992; 77(1): 65-78. 
7. Frone MR, Russell M, Cooper ML. Prevalence of work-family conflict: Are work and family boundaries asymmetrically permeable? J Organ Behav. 1992; 13(7):723-729.

8. Kahn RL, Wolfe DM, Quinn RP, Snoek JD, Rosenthal RA. Organizational stress: Studies in role conflict and ambiguity. New York: Wiley; 1964.

9. Aryee S, Field D, Luk V. A cross-cultural test of a model of the work-family interface. J Manage. 1999. 25(4): 491-511.

10. Michel JS, Kotrba LM, Mitchelson JK, Clark MA, Baltes BB. Antecedents of work-family conflict: A metaanalytic review. J Organ Behav. 2011; 32(5): 689-725.

11. Kinnunen U, Feldt T, Geurts S, Pulkkinen L. Types of work-family interface: Well-being correlates of negative and positive spillover between work and family. Scand J Psychol. 2006; 47: 49-162.

12. Van Hooff M, Geurts S, Kompier M, Taris T, Houtman I, Van den Heuvel F. Disentangling the causal relationships between work-home interference and employee health. Scand J Work Environ Health. 2005; 31(1): 15-29.

13. Boyar SL, Maertz Jr CP., Pearson AW, Keough S. Work-family conflict: A model of linkages between work and family domain variables and turnover intentions. Journal of Managerial Issues. 2003; 15(2): 175-190.

14. Marín M, Infante E, Rivero M. Presiones internas del ámbito laboral y/o familiar como antecedentes del conflicto trabajo-familia. Revista de Psicología Social. 2002; 17(1): 103-112.

15. Anderson SE, Coffey BS, Byerly RT. Formal organizational initiatives and informal workplace practices: Links to work-family conflict and job-related outcomes. J Manage. 2002; 28(6): 787-810.

16. Luk DM, Shaffer MA. Work and family domain stressors and support: Within-and cross-domain influences on work-family conflict. J Occup Organ Psychol. 2005; 78(4): 489-508.

17. Ford MT, Heinen BA, Langkamer KL. Work and family satisfaction and conflict: A meta-analysis of cross-domain relations. J Appl Psychol. 2007; 92(1): 57-80.

18. Boyar SL, Mosley Jr. DC. The relationship between core self-evaluations and work and family satisfaction: The mediating role of work-family conflict and facilitation. J Vocat Behav. 2007; 71(2): 265-281.

19. Graves LM, Ohlott PJ, Ruderman MN. Commitment to Family Roles: Effects on Managers' Attitudes and Performance. J Appl Psychol. 2007; 92(1): 44-56.

20. Lapierre LM, Allen TD. Work-supportive family, family-supportive supervision, use of organizational benefits, and problem-focused coping: Implications for work-family conflict and employee well-being. J Occup Health Psychol. 2006; 11(2): 169-181.

21. Mauno S, Kinnunen U, Pyykkö M. Does work-family conflict mediate the relationship between workfamily cultura and self-reported distress? Evidence from five Finnish organizations. J Occup Organ Psychol. 2005; 78(4): 509-530.

22. Hill EJ. Work-family facilitation and conflict, working fathers and mothers, work-family stressors and support. J Fam Issues. 2005; 26(6): 793-819.

23. Goff SJ, Mount MK, Jamison RL. Employer supported child care, work-family conflict and abseentism: a field study. Pers Psychol. 1990; 43(4):7 93-809.

24. Kossek EE, Pichler S, Bodner T,Hammer LB. Workplace social support and work-family conflict: A meta-analysis clarifying the influence of general and work-family-specific supervisor and organizational support. Pers Psychol. 2011; 64(2): 289-313.

25. Hammer L, Kossek E, Yragui N, Bodner T, Hanson G. Development and validation of a multidimensional measure of family-supportive supervisor behaviors. J Manage. 2009; 35(4): 837-856.

26. Aryee S, Luk V, Leung A, Lo S. Role stressors, interrole conflict, and well-being: The moderating influence of spousal support and coping behaviors among employed parents in Hong Kong. J Vocat Behav. 1999; 54(2): 259-278.

27. Noor NM. The moderating effect of spouse support of the relationship between work variables and women's work-family conflict. Psychologia. 2002; 45(1):12-23.

28. Frone MR, Rice RW. Work-family conflict: The effect of job and family involvement. J Occup Behav. 1987; 8: 45-53.

29. Kinnunen U, Vermulst A, Mäkikangas A. Work-family conflict and its relations to well-being: The role of personality as a moderating factor. Pers Individ Dif. 2003; 35(7): 1669-1683.

30. Rantanen M, Mauno S, Kinnunen U, Rantanen J. Do individual coping strategies help or harm in the work-family conflict situation? Examining coping as a moderator between work-family conflict and well-being. Int J Stress Manag. 2011; 18(1): 24-48

31. Geurts S, Sonnentag S. Recovery as an explanatory mechanism in the relation between acute stress reactions and chronic health impairment. Scand J Work Environ Health. 2006; 32(6): 482-492. 
32. Sonnentag S, Fritz C. The Recovery Experience Questionnaire: Development and Validation of a Measure for Assessing Recuperation and Unwinding from Work. J Occup Health Psychol. 2007; 12(3): 204-221.

33. Jansen NW, Kant I, Kristensen TS, Nijhuis FJ. Antecedents and consequences of work-family conflict: A prospective cohort study. J Occup Environ Med. 2003; 45(5): 479-491.

34. Taris T, Beckers D, Verhoeven L, Geurts S, Kompier M, Van der Linden D. Recovery opportunities, workhome interference, and well-being among managers. Eur J Work Organ Psychol. 2006; 15(2): 139-157.

35. Dembe A.E. The changing nature of office work: Effects on repetitive strain injuries. Occup Med. 1999; 14(1): 61-72.

36. Fritz C, Sonnentag S. Recovery, well-being, and performance-related outcomes: The role of workload and vacation experiences. J Appl Psychol. 2006; 91(4): 936-945.

37. Sonnentag S. Recovery, work engagement, and proactive behavior: A new look at the interface between nonwork and work. J Appl Psychol. 2003; 88(3): 518-528.

38. Sonnentag S, Bayer U. Switching off mentally: Predictors and consequences of psychological detachment from work during off-job time. J Occup Health Psychol. 2005; 10(4): 393-414.

39. Sonnentag S, Binnewies C, Mojza EJ. Did you have a nice evening? A day-level study on recovery experiences, sleep, and affect. J Appl Psychol. 2008; 93(3): 674-684.

40. Moreno-Jiménez B, Mayo M, Sanz-Vergel AI, Geurts S, Rodríguez-Muñoz A, Garrosa E. Effects of workfamily conflict on employee's well-being: The moderating role of recovery strategies. J Occup Health Psychol. 2009; 14(4): 427-440.

41. Sanz-Vergel AI, Demerouti E, Mayo M, Moreno-Jiménez B. Work-home interaction and psychological strain: The moderating role of sleep quality. Appl Psychol-Int Rev. 2011; 60(2): 210-230.

42. Hobfoll SE. Conservation of resources: A new attempt at conceptualizing stress. Am Psychol. 1989; 44(3): 513-524.

43. Meijman TF, Mulder G. Psychological aspects of workload. En Drenth PJD, Thierry H. (Eds.), Handbook of work and organizational psychology (Vol. 2: Work psychology, pp. 5-33). Hove, Inglaterra: Psychology Press; 1998.

44. Covey S, Merrill R, Merrill R. First things first. Nueva York: Simon \& Schuster; 1994.

45. Kareaga, A. Afrontamiento del estrés en las organizaciones: Un programa de manejo a nivel individual/ grupal. Revista de Psicología del Trabajo y de las Organizaciones. 2004; 20(1):77-93.

ІІІ!| 